\title{
Label-free detection of nucleic acids and proteins using impedance spectroscopy and surface plasmon resonance
}

\author{
M. Riedel, F. Heinrich, F. Lisdat \\ Biosystems Technology, Institute of Applied Life Sciences, Technical University Wildau, \\ Hochschulring 1, 15745 Wildau, \\ flisdat@th-wildau.de
}

\begin{abstract}
Label-free technologies have gained increasing interest in sensing. The presentation focuses on the use of impedance measurements for the analytical detection of nucleic acids including abasic DNA and DNA-binding proteins. It will show how pitfalls can be avoided by elimination of drift phenomena. Furthermore, it will illustrate the usefulness of surface plasmon resonance in the detection of conformational changes and the discrimination of two rather similar proteins.
\end{abstract}

Key words: impedance spectroscopy, mismatch, abasic DNA, G-quadruplex, ricin, SPR

\begin{abstract}
Introduction
Label free technologies such as impedance spectroscopy or surface plasmon resonance are not only useful during the development of sensors since they allow to follow the different steps of surface modification during preparation, but they have increasingly shown the potential for their use as an analytical tool [1]. In addition, the combination of impedimetric measurements and SPR can give valuable hints for the behaviour of molecules on the sensing surface and thus, can help to understand sensing effects.
\end{abstract}

The presentation will show the different information one can get from the impedimetric detection of a hybridization event in comparison to a SPR detection and how this can help to understand the behaviour of longer DNA strands binding to the electrode surface. Abasic DNA is a strand at which a complete base is missing. The reason for this can be various including radiation, alkylating agents or enzyme action [2]. Little is known so fare on the binding behaviour of this type of DNA to immobilized capture strands and how such duplexes will influence the surface properties.

In addition, it will be shown that conformational changes such as the formation of secondary structures of nucleic acids can be detected by a proper design of the nucleic acid sequence to be immobilized on the sensor as recognition element. Thus, sensitivity limitations can be overcome.

Finally, another aspect will be treated since often the discrimination of similar proteins is not a trivial task - even when antibodies are available for them. Immobilization of the capture antibody in a 3D matrix allows to exploit steric effects in order to analyze two different analytes in one sample [3].

\section{Impedimetric DNA and protein detection}

Impedance spectroscopy can be shown to allow the analytical detection of SsDNA by hybridization to a complementary capture strand on the electrode surface [4]. This is mainly based on electrostatic effects since here the highly charged redox couple ferri/ferrocyanide is used. Different factors influence the detection capabilities, for example the density of immobilized capture probes and the buffer concentration. In real analytical situations often the amount of DNA is rather small and thus, protocols such as PCR or isothermal procedures are used in order to amplify the nucleic acids. This will results however, in molecules, which are much longer than the immobilized capture probe. Here the combined measurement of SPR and impedance can help to clarify the influence of the mass which is 
bound and the number of molecules binding to the sensing surface on the impedimetric signal generation.

Time dependent changes in impedance have sometimes reported in the literature. Here care has to be taken in the preparation of the sensing electrode in order to get stable signals in the situation of an unbound ss-capture probe. Furthermore, the denaturation protocol needs to be optimized to avoid long-term drifts.

On this basis the analysis of the binding of abasic ssDNA to the immobilized capture probe is feasible. Here it can be seen that the impedance changes after contact of the sensing surface with the nucleic acid are significantly smaller compared to a fullmatch DNA. This is illustrated in Fig. 1. for high concentrations of the nucleic acids during hybridization. Furthermore, the position of the abasic site within the formed double strand has a significant influence on the binding kinetics, but also on the overall DNA amount bound. With the impedance detection alone the effects on the recognition surface cannot be understood. Comparative measurements by SPR analyzing the mass deposition during the hybridization help to understand that not only the hybridization efficiency is influenced, but also indicate that different structures of the DNA layer result by abasic DNA binding.

Finally, it can be shown that even protein binding to DNA is feasible to be detected impedimetrically. This can be demonstrated even for sequence-specific binding such as with a transcription factor in a concentration dependent manner.

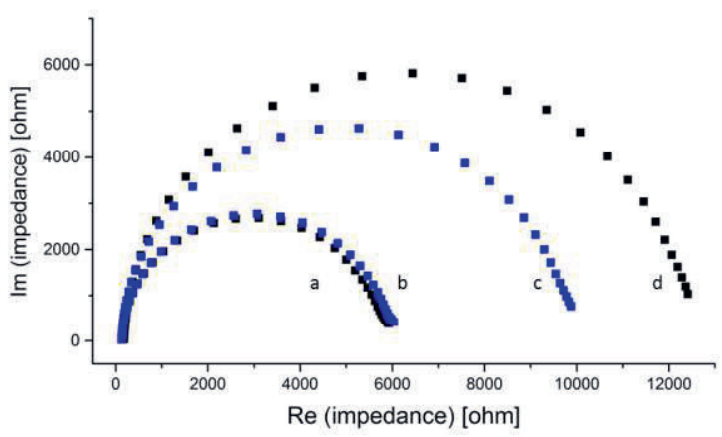

Fig. 1. Impedance spectra measured for a gold electrode modified with ssDNA $(a, b)$ and after hybridization with a fullmatch DNA (d) or an abasic DNA (c) with the abasic site located close to the electrode. Phosphate buffer $\mathrm{pH} 7$ ferri-/ferrocyanide $2 \mathrm{mM}$, frequency range $1 \mathrm{~Hz}-10 \mathrm{kHz}$ Hybridisation with $10 \mu \mathrm{M}$ nucleic acid in each case.

\section{DNA conformational changes and} simultaneous analysis of two proteins

SPR cannot only help to analyze the amount of bound material during a certain contact time but can also be applied as analytical tool. Conformational changes of biomolecules on a surface are not easy to detect since the overall molecule amount is not changing by the process. Thus, for example the formation of a G-quadruplex in a G-rich sequence cannot be resolved in a SPR experiment with an immobilized oligonucleotide sequence. However, the situation on the sensor surface can drastically be improved when the design of the recognition element is changed in order to enforce larger alterations in the optical density near the sensor surface. In such a situation the on-line detection of the secondary structure formation becomes feasible and can be studied in dependence on the solution composition.

Another aspect is related to the detection of protein binding for analytical purpose when two rather similar proteins have to be analysed which cannot be clearly distinguished in an ELISA format. Even when the SPR analysis of antibody affinity show that no clear discrimination in the recognition process occurs, the online binding detection capabilities of SPR can be applied in a two-step injection procedure with normalization allowing a clear quantification of both proteins.

\section{References}

[1] M. Riedel, F. Lisdat, "Biosensorial Application of Impedance Spectroscopy with Focus on DNA Detection", Springer Series on Chemical Sensors and Biosensors, doi: 10.1007/5346_2017_18, Springer (2017)

[2] J. Lhomme, JF.Constant, M. Demeunynck, Biopolymers 52(2) 1999 65-83, doi: 10.1002/1097-0282(1999)52:2<65::AIDBIP1>3.3.CO;2-L

[3] D. Stern, D. Pauly, M. Zydek, C. Müller, M. A. Avondet, S. Worbs, F. Lisdat, M. Dorner and B. G. Dorner, Biosensors \& Bioelectronics 2016, Vol. 78, p. 111, doi:10.1016/j.bios.2015.11.020

[4] M. Riedel, J. Kartchemnik, M. Schöning, F. Lisdat, Anal. Chem. 2014, Vol.86 p. 7867, doi: 10.1021/ac501800q 